\title{
Child and adolescent mental health problems in primary care
}

\author{
Tami Kramer \& M. Elena Garralda
}

Epidemiological research has revealed that psychiatric disorders in children and adolescents are common, persistent and handicapping. Only 1 in 10 of those with a disorder is seen in specialist mental health services. However, the majority of children and adolescents see their general practitioner (GP) every year. Although the majority present with physical complaints, there are indications that rates of psychiatric disorder in those attending are increased and that psychiatric disorder is associated with increased consulting. These findings raise questions about the role of primary care in the promotion and management of mental health in young people.

Research evidence of the prevalence, patterns and detection of psychiatric disorder in primary care has been accumulating. Factors affecting consultation and referral to specialist services have received attention. Here, we will summarise this evidence before discussing studies of interventions within primary care. Implications for service delivery and future directions for research will be discussed. This review will focus mainly on studies of children and adolescents in primary care within the UK; however, where suitable data are not available, relevant studies of other countries, settings or age groups will be cited.

\section{Prevalence, pattern and detection of psychiatric disorder in primary care}

During any one year, the majority of children and adolescents attend their GP. Two to five per cent present with emotional or behavioural problems, while the rest present with physical complaints. Studies of attenders (Garralda \& Bailey, 1986; Kramer \& Garralda, 1998) using standardised diagnostic criteria reveal high rates of psychiatric disorders ( 1 in 4 of 7 - to 12-year-olds; 4 in 10 of 13- to 16-year-olds). Although the range of common psychiatric disorders is seen, there is specificity in the type of disorder seen in surgery attenders. There is an excess of oppositional defiant disorders seen in pre-school children (Lavigne et al, 1993) and an excess of emotional disorders over conduct disorders in school children and adolescents. As well as suffering and impairment, psychiatric disorder in adolescent attenders is linked with increased frequency and intensity of physical symptoms, impairment from these physical symptoms and increased exposure to illicit drugs (Kramer \& Garralda, 1998). Furthermore, psychiatric disorder is associated with increased health service utilisation. Frequent GP attendance has been linked with psychiatric disorder in school children (Bowman \& Garralda, 1993) and mood disturbance in adolescent girls (Moncket al, 1994).

General practitioner recognition of psychiatric disorder in children is limited, with wide variation between practitioners. Their assessments have moderate sensitivity and high specificity. Recognition is better in children or adolescents with more severe disturbance and in those with stressful family situations. However, it is likely that those who are less disturbed, or identified early, may actually respond better to less intensive interventions in primary care.

Tami Kramer is a Clinical Lecturer in Child and Adolescent Psychiatry at Imperial College School of Medicine (St Mary's Campus, Norfolk Place, Paddington, London W2 1PG). She has spent the past five years researching the contribution of psychiatric disorder to adolescent attendance in primary care and appropriate interventions. Elena Garralda is Professor of Child and Adolescent Psychiatry at Imperial College School of Medicine. She has a long-standing research interest in the interface between child and adolescent psychiatry and primary care. 
Box 1. Prevalence and patterns of psychiatric disorder in primary care

The majority of children and adolescents attend their GP in any one year

Two to five per cent present with emotional or behavioural problems

Rates of psychiatric disorders in attenders is high: 1 in 4 of 7-12 year olds; 4 in 10 of 13-16 year olds

Types of disorder in attenders

Pre-school children: oppositional defiant disorders

School children and adolescents: emotional disorders

Psychiatric disorder is linked with increased physical symptoms, health service utilisation and health risks

\section{Consultation and perception of need}

Most ill health episodes do not lead to consultation, and most primary care consultations are ostensibly for physical complaints. However, increased rates of psychiatric disorder in primary care attenders over general population rates (for all ages) suggest that the presence of psychiatric disorder increases the likelihood of consultation. Although only $2-5 \%$ of consultors present with emotional or behavioural complaints, there are indications that consultors are aware that psychological factors contribute to their symptoms. One-third of adolescents with undetected psychiatric disorder who consulted for physical complaints recognised that their physical symptoms were worse with stress. In a study of 8-14 year olds attending their GP in London (Evans \& Brown, 1993), 13\% were found to be at high risk for psychiatric disorder, and $12 \%$ of parents felt that their child would benefit from individual or family counselling. The GPs were certain that child mental health services were needed for $5 \%$ of children attending and were unsure for a further $10 \%$. However, only $3 \%$ of consulting children and adolescents had been in contact with mental health clinics in the previous year. These findings suggest that although the majority of consultations are for physical complaints, a number of parents are able to recognise their children's currently undiagnosed emotional and behavioural needs.

\section{Referral to specialist mental health services}

The factors that determine referral to specialist mental health services by GPs are not well understood. Doctors' recognition of disturbance is as important as parental attitudes towards children's problems, since doctors are often responsible for seeking help. Referral is linked with more severe disturbance and male gender and, consistent with the increase of boys among referred children, an excess of antisocial symptoms (Garralda \& Bailey, 1988). The severity of referred disturbed children's difficulties appears often to be part of multiple psychosocial family stress, including unemployment, marital and mental health problems in the mothers, low support from their families, socioeconomic disadvantage and high levels of stress felt by the parents in relation to parenting. Children are unlikely to be referred unless they present with overt psychological symptoms. Maternal request for referral is an important determinant in many cases (Garralda \& Bailey, 1988). For parents requesting referral for obviously seriously disturbed children within difficult social and socio-economic circumstances, referral to a specialist mental health service is appropriate. However, the role of the GP in detecting and managing cases with less severe or less complex psychiatric disorders, not overtly presented, and currently largely unrecognised, has increasingly come into focus.

\section{Interventions}

GPs are often trusted by families and are therefore in a position to provide them with information, support and advice. They may thus also be in a good position to implement preventive interventions. GP contact with children and adolescents suffering with established psychiatric disorders provides a potential opportunity for early identification and treatment of disorder, in addition to recognition of disorder requiring referral to specialist services. Advantages of the provision of services within primary care include: (a) the GP often already has knowledge of a family's circumstances over time; (b) general practice is often seen as less stigmatising than psychiatric services; and (c) patients may prefer this option. Studies within adult mental health have 
shown that primary care interventions are feasible and effective. Controlled studies of interventions with children and adolescents are methodologically difficult to implement, but growing interest has led to a number of interesting initiatives. Additionally, evidence is accumulating with regard to the potential contribution of other members within the primary care team to interventions for children and families.

\section{Screening and detection}

The prospect of receiving treatment for psychiatric disturbance is currently limited by low rates of detection within primary care. A number of approaches have tried to improve recognition. Questionnaires have been used as screening tools and although they achieve identification, their contribution towards helping clinicians attend to difficulty remains unclear. Opening questions by clinicians can be productive in identifying actual or associated reasons for consulting as opposed to ostensible reasons.

Bernard et al (1999) developed a single-session training package for GP trainees aimed at improving knowledge, assessment, identification and management of child and adolescent mental health (CAMH) problems. The training included preparatory reading (Hughes et al, 1994) and a teaching session (with problem-solving exercises, role-play and video vignettes). The trainees demonstrated improved selfperceived competence, knowledge and recognition of disorder.

In addition to direct identification of mental health problems in children, exploration of other psychosocial and health risks can effectively alert the GP to the presence of child psychiatric disorder. Among frequently attending school children, this may include children with persistent functional symptoms, those who are markedly handicapped or impeded by physical complaints, those with peer relationship difficulties and those with mothers in poor mental health (Garralda et al, 1999).

Two reports have outlined the results of screening adolescents for mental health problems within primary care. Donovan \& McCarthy (1988) invited all 16-17 year olds registered with their practice to attend to discuss "any medical or general problems". Over $50 \%$ attended after one letter. Depression, acne and obesity were the most common problems reported. Westman \& Garralda (1996) investigated the feasibility of inviting 14-15 year olds to a health promotion clinic to explore mental and physical health and to identify those at high risk of depression. Overall, attendance rates were low (22\%), but higher if adolescents were invited to attend without their parents. A high proportion had probable previously undetected psychiatric disorder, with depression being the most common diagnosis. Using community prevalence rates, the authors calculated that the clinic probably attracted all registered adolescents with depression. Most had attended their GP in the previous year, suggesting that their depression could have been detected when they attended and that separate screening clinics may not be cost-effective for adolescent depression.

\section{Interventions by the GP}

Although there are few controlled studies of interventions by GPs, interesting initiatives that could be offered have been described. These include interventions developed by GPs and adaptations of specialist skills by CAMH workers for use in primary care.

Cullen (1976), a GP in Australia, documented early and long-term follow-up effects of a preventive intervention. The author saw mothers of 124 children for 12 20-30-minute sessions over the first five years of the child's life. Child-rearing concepts and attitudes were explored. At age 6 years, experimental children were found to have fewer fears, sleep disorders, eating problems and less aggression. They also revealed significantly more positive and negative feelings towards their mothers. Thus, the intervention demonstrated the influence of a doctor on parental actions and children's behaviour and feelings. Follow-up of these children 20 years later (Cullen \& Cullen, 1996) suggested longlasting favourable effects for the experimental women, who reported fewer neurotic symptoms, more university qualifications and less obesity. Early pioneering efforts of this sort represent a good model on which to base more extensive and rigorous evaluation.

More recently, attempts have been made to adapt for use in primary care management techniques employed within secondary CAMH services. Coverly et al (1995) developed a structured single-session psychiatric intervention for mothers of school children attending primary care who were found from research interviews to have mental health problems. The intervention aimed to boost parental confidence and provide effective parent management. Nearly two-thirds of the mothers found the intervention markedly useful or extremely useful at 3- and 18-24-month follow-ups. Parents reported a decrease in problem severity and in their level of concern; confidence in managing the problem was increased. The behavioural questionnaire scores, however, did not show a corresponding reduction. A trend towards reduced surgery attendance occurred following the intervention in parents who felt that they had benefited. These findings are in 
line with those of Finney et al (1991) from the USA. In this study, children with common behavioural, school, toilet and psychosomatic problems were offered 1-6 sessions within primary care. Parental outcome and behaviour checklist ratings showed improvement or resolution in $74 \%$ of cases with high satisfaction. Use of medical services in the following year, especially primary care visits, decreased when compared with a matched control group. These mainly open, uncontrolled studies are promising and call for more rigorous evaluation.

Pilot interventions (Westman \& Garralda, 1996; Kramer \& Garralda: details available from the author upon request) offering brief psychotherapeutic interventions (4-6 sessions) to adolescents with depression and anxiety attending primary care have demonstrated that uptake of treatment by this age group is poor. This suggests that an intervention offered by a GP within the index consultation may be the most useful alternative to develop and evaluate further.

The role of the GP involves a relationship with the whole family, and the GP often has knowledge of events leading to family crises. Thus, the GP is in a good position to address children's difficulties within the family context. Senior (1994) described the development of a family therapy clinic within a general practice aimed at difficult families who had not responded to traditional interventions or who were in crisis. Graham et al (1992) evaluated the work with 19 families. Referrers found the clinic helpful. Half of the adult attenders responded and were positive about the therapy's effectiveness and acceptability. Although almost all responders felt that it was easier to attend a clinic at the practice than at a hospital or child guidance clinic, this intervention is likely to be costly. It involves the time of a multi-disciplinary team of highly trained professionals (including GPs, social workers and family therapists) initially supervised regularly by a consultant psychiatrist. Since attendance rates may not have been substantially improved, it seems questionable whether these complex interventions should be systematically offered in primary care.

In addition to their role in managing more typical emotional and behavioural difficulties, primary care professionals have an important role in managing psychosomatic problems, where a holistic approach, combining attention to physical and emotional aspects, is particularly appropriate.

\section{Interventions by the primary care team}

The role of the primary care team in preventing and treating mental health problems in children may be broader and more intensive than the GP's and include interventions with children and mothers. Collaborative support from specialist mental health services, paediatric services and public health services is likely to be needed to organise programmes of primary or early secondary prevention, focusing on high-risk populations.

The role of the primary care team in interventions for pre-school children, whose main environment is the home, is particularly important since older children can receive interventions in other settings, for example, school. Research from the USA (Olds et $a l, 1997)$ has demonstrated the benefits of preventive home visiting. In this 15-year follow-up of a randomised controlled trial of home visiting for poor mothers during pregnancy and up to the child's second birthday, there were significant psychosocial benefits for mothers and significantly less abuse and neglect of children.

There is evidence that early community treatment of mothers with post-natal depression (with counselling sessions delivered by child health nurses) can lead to significantly greater rates of recovery than in a control group of mothers (Holden et al, 1989; Wickberg \& Hwang, 1996). Since these interventions aim to prevent the negative effects of post-natal depression on the mother-infant relationship and infant emotional and cognitive development, future investigations should include measures of outcome for the children.

Cox (1993) summarises the approaches implemented by health visitors to deal with emotional and behavioural problems in young children. He concludes that systematic evaluations using control groups have all been equivocal in terms of benefits to maternal mental health, mothers' reported ability to manage emotional and behavioural problems, and the level of behavioural problems in children. Tsiantis et al (1996) have described a programme to train primary health care workers in the promotion of children's early psychosocial development through contact with mothers from conception until their child is 2 years old. It was planned to be evaluated across various European countries.

The prevalence of oppositional defiant disorders in pre-school primary care attenders (Lavigne et $a l, 1993$ ) and evidence for effective prevention suggest that greater involvement of primary care services may be beneficial. This is important since oppositional disorders may be precursors of conduct disorders and in view of current concern about high rates of and difficulty treating conduct disorders. Webster Stratton (1998) implemented a parenting programme in the USA with 394 parents of 4 year olds attending Head Start (a social and cognitive stimulation programme for low-income 
families). The programme aimed to prevent the onset of oppositional defiant and conduct disorders and covered how to play with and help your child learn, use of praise, limit setting and problem-solving. A randomised controlled trial demonstrated significant differences in those receiving the intervention, most of which were maintained 1 year later. Significant differences were found in children (increased social competence, fewer conduct problems, less non-compliance and less negative affect) and mothers (more positive competent parenting). As an 8-9-session group programme it could feasibly be implemented by a trained nurse within primary care. Alternatively, primary care could contribute by identifying high-risk families and facilitating referral to existing community programs of this type.

\section{Provision of services for CAMH in primary care}

There is little published information on what is being provided for CAMH within primary care or within liaison schemes with child psychiatrists.

Adult psychiatry has developed and evaluated various models of joint working at the interface between primary care and secondary mental health services, which raise discussion relevant to child psychiatry. Mitchell (1985) described these models, which included: (a) coordinated home visits by GP and mental health worker; (b) shift of psychiatric clinics to health centres or surgeries; (c) visits to health centres to see selected patients; (d) group discussions with primary care staff; and (e) conjoint consultations.

A decade later, Gask et al (1997) reviewed developments in adult service provision at the interface between primary and secondary care. Sectorised community mental health teams (CMHTs) have led to varying patterns of integration with primary care. The shifted out-patient clinic, where psychiatrists operate clinics within primary care, is widely used. Many primary care practices have mental health professionals attached (e.g. community psychiatric nurses, psychologists or counsellors). Consultation-liaison, which emphasises creating links between primary care and psychiatric staff, has been developed in combination with the developments above. Consultation-liaison aims to: (a) reduce referrals of milder disorders; (b) encourage referral of serious mental illness; and (c) enhance GPs' skills in the detection and management of mental illness. It involves regular meetings between the psychiatrist, GP and members of the primary care team, with discussion of possible referrals and feedback after cases are assessed.

In many ways, multi-disciplinary child mental health teams resemble CMHTs. However, the ratio per population is much lower, which limits the possibility of offering shifted out-patient clinics, attached mental health professionals or increased consultation-liaison, without considerable expansion of services. Furthermore, the aims of consultationliaison would need modification. Within child and adolescent psychiatry, the distinction between 'milder disorders' and 'serious mental illness' is more difficult to define, since serious problems are often not qualitatively different from milder ones. The large numbers of young people with disorders make it unlikely that child psychiatrists could ever attempt to be directly involved with the majority of cases. Therefore, an important task is the definition of cases needing multi-disciplinary assessment and treatment $v$. those needing intervention from a single-handed skilled professional. Enhanced GP skills in detection and management of disorders, are also necessary for children and adolescents within any future models of service delivery. Consultation-liaison provides one method of enhancing this - it would at least improve communication and understanding of what primary care workers and child psychiatrists need from each other, and might facilitate engagement of families that are difficult to refer.

The Health Advisory Service (HAS) report (1995) recognised the need for the development of provision for the mental health of children and adolescents within first-line services. The report recommended the development of tiered services with the introduction of primary mental health workers (PMHWs). They would assess and treat some individuals within the first-line services, educate and support other professionals within these services and improve links between primary and specialist services. As members of the mental health team working within primary care, they would incorporate elements of the shifted out-patient clinic, the attached mental health professional and consultation-liaison.

The HAS recommendations are open to a wide range of interpretations and there is still little published material on the implementation. It is essential that new developments are evaluated. Gask et al (1997) detail important areas for evaluation that are equally applicable to children and adolescents and include: the impact of service developments on referral patterns (numbers of cases, types of cases and appropriateness of referrals), changes in GPs' ability to detect and manage cases which are not referred, and quality and outcome of different models of care (including cost-effectiveness). 
In addition, if services are to be developed in a tiered way, as recommended, a number of important areas still require examination:

(a) Clarification of which cases should be seen at which level: this is likely to require the development of referral protocols.

(b) Who is best equipped to take up the role of the primary mental health worker? Level of experience and professional background need examination. Experience needed to provide first-line treatment interventions may be different from that needed to select those cases that require initial referral to a specialist centre.

(c) Development of first-line services may not necessarily decrease the number of appropriate referrals to specialist services. More cases in need of referral may be detected and secondary services will need to accommodate this.

(d) In the current situation of high levels of unmet need and limited resources, priorities need to be set in terms of a balance between preventive work in high-risk groups (with possible long-term benefits) and treatment for clinical cases.

Two published studies have begun to evaluate new developments. Neira-Munoz \& Ward (1998) reported a controlled pilot study of a liaison service delivered by a PMHW. The role of the PMHW included: (a) deciding with the primary care team on appropriate interventions to be delivered within primary care; (b) assessment of appropriateness of referrals; and (c) offering a clinical service within primary care. Most primary care professionals found the liaison clinics of value, many reported an increase in knowledge and skills, and the service resulted in fewer referrals to the specialist service. These findings need replication in a larger study with more discussion of the professional background and level of experience of the PMHW.

Davis \& Spurr (1998) evaluated a parent-counselling programme carried out by health visitors and clinical medical officers for parents of pre-school children. Most referrals came from health visitors. A quarter did not take up the intervention despite it being offered at home. The authors point to positive outcomes in terms of parental well-being and child behavioural problems, but methodological problems limit the significance of the findings. The initial assessment for this intervention was carried out jointly with the supervisor, suggesting the need for involvement of an experienced clinician at this stage.

One of the obstacles to developing a comprehensive approach for mental health within primary services has been the need for specialist services to link with a large number of individual general practices. The recent shift of the purchasing function to primary care groups (PCGs) provides a new opportunity. Since each specialist service liaises with a few PCGs there is scope for the development of initiatives within this structure. Specialist mental health services and PCGs can develop a joint set of priorities for the development of primary care $\mathrm{CAMH}$ services based on local need and resources. The literature discussed above suggests that this should include a combination of preventive interventions for high-risk mothers and brief treatments offered within primary care for less severe and complicated problems in the child. The former may focus particularly on support for mothers, treatment of post-natal depression and management of behavioural problems in pre-schoolers. The latter may address the milder emotional and behavioural problems in school children and depressive disorders in adolescents. PMHWs could offer PCGs a combination of group treatment programmes (e.g. parenting programmes for oppositional children), brief treatments within primary care, and training and support of other members of the team. They should also work in conjunction with secondary services to aid implementation of referral protocols. Specialist mental health services need to be involved in developing and supporting these initiatives.

Box 2. Areas for future work

Improved detection of CAMH problems in primary care

Controlled primary care interventions for:

- Pre-school children with oppositional defiant disorders

- School children with emotional/internalising disorders (including psychosomatic symptoms)

- Adolescents with depressive and anxiety disorders

Service development and monitoring including the following areas:

- Shifted out-patient clinics

- Consultation liaison

- Referral protocols for referral to secondary care

- Service development conjointly with PCGs 


\section{Conclusions}

Clinicians and health service planners face a difficult challenge over coming years. How can evidence-based treatments be adapted to meet the mental health needs of children and adolescents with disorders attending primary care? Despite high levels of psychiatric disorder in attenders, recognition of disorders and access to help are very limited. Since resources for treatment are finite, the first challenge may be to prevent development of severe disorders and increase early recognition. Specific disorders could appropriately be targeted. Increased availability of help within primary care requires the support and education of professionals within that setting. It may also require expansion of services provided by a professional intermediate to primary care and specialist services, such as the PMHW.

The challenge for researchers in this area is also complex. There is a need for further studies of effects of the following: preventive interventions delivered by ordinary services; effects of detection of covert disorders and early intervention on short- and longterm outcome; controlled studies of interventions within the primary care setting; and monitoring the effects of changes in service delivery on patients, on other health service sectors and on other agencies.

\section{References}

Bernard, P., Garralda, M. E., Hughes, T., et al (1999) Evaluation of a teaching package in adolescent psychiatry for general practice registrars. Education for General Practice, 10, 21-28.

Bowman, F. M. \& Garralda, M. E. (1993) Psychiatric morbidity among children who are frequent attenders in general practice. British Journal of General Practice, 43, 6-9.

Coverly, C., Garralda, M. E. \& Bowman, F. (1995) Psychiatric intervention in primary care for mothers whose schoolchildren have psychiatric disorder. British Journal of General Practice, 45, 235-237.

Cox, A. (1993) Preventative aspects of child psychiatry. In Managing Children with Psychiatric Problems (ed. E. Garralda), pp. 179-207. London: BMJ Publishing Group.

Cullen, K. J. (1976) A six-year controlled trial of prevention of children's behaviour disorders. Journal of Pediatrics, $\mathbf{8 8}$, 662-666.

- \& Cullen, A. M. (1996) Long-term follow-up of the Busselton six-year controlled trial of prevention of children's behaviour disorders. Journal of Paediatrics, 129, 136-139.

Davis, H. \& Spurr, P. (1998) Parent Counselling: An evaluation of a community child mental health service. Journal of Child Psychology and Psychiatry, 39, 365-376.

Donovan, C. F. \& McCarthy, S. (1988) Is there a place for adolescent screening in general practice? Health Trends, 20, 64-66.

Evans, S. \& Brown, R. (1993) Perception of need for child psychiatry services among parents and GPs. Health Trends, $25,53-56$.
Finney, J. W., Riley, A. W. \& Cataldo, M. F. (1991) Psychology in primary health care: effects of brief targeted therapy on children's medical care utilization. Journal of Paediatric Psychology, 16, 447-461.

Garralda, M. E. \& Bailey, D. (1986) Children with psychiatric disorders in primary care. Journal of Child Psychology and Psychiatry, 27, 611-624.

— \& Bailey, D. (1988) Child and family factors associated with referral child psychiatrists. British Journal of Psychiatry, 153, 81-89.

—, Bowman, F. M. \& Mandalia, S. (1999) Children with psychiatric disorders who are frequent attenders to primary care. European Child and Adolescent Psychiatry, 8, $34-44$.

Gask, L. Sibbald, B. \& Creed, F. (1997) Evaluating models of working at the interface between mental health services and primary care. British Journal of Psychiatry, 170, 6-11.

Graham, H., Senior, R., Lazarus, M., et al (1992) Family therapy in general practice: views of referrers and clients. British Journal of General Practice, 42, 25-28.

Health Advisory Service (1995) Child and Adolescent Mental Health Services: Together We Stand. London: HMSO.

Holden, J. M., Sagoysky, R. \& Cox, J. L. (1989) Counselling in a general practice setting: controlled study of health visitor intervention in treatment of postnatal depression. British Medical Journal, 298, 223-226.

Hughes, T, Garralda, M. E \& Tylee, A. (1994) Child Mental Health Problems. London: St Mary's Hospital Medical School.

Kramer, T. \& Garralda, M. E. (1998) Psychiatric disorders in adolescents in primary care. British Journal of Psychiatry, $173,508-513$.

Lavigne, J. V., Binns, H. J., Christoffel, K. K., et al (1993) Behavioural and emotional problems among pre-school children in paediatric primary care: prevalence and paediatricians' recognition. Pediatrics, 91, 649-655.

Mitchell, A. R. K. (1985) Psychiatrists in primary health care settings. British Journal of Psychiatry, 147, 371-179.

Monck, E., Graham P., Richman, N., et al (1994) Adolescent girls I. Self-reported mood disturbance in a community population. British Journal of Psychiatry, 165, 760-769.

Neira-Munoz, E. \& Ward, D. (1998) Side by side. Health Service Journal, 108, 26-27.

Olds, D. L., Eckenrade, J., Henderson, C. R., et al (1997) Long-term effects of home visitation on maternal life course and child abuse and neglect. Journal of the American Medical Association, 278, 637-643.

Senior, R. (1994) Family therapy in general practice: "We have a clinic here on Friday afternoon...". Journal of Family Therapy, 16, 313-327.

Tsiantis, J., Dragonas, T., Cox, A., et al (1996) Promotion of children's early psychosocial development through primary health care services. Paediatric and Perinatal Epidemiology, 10, 339-354.

Webster Stratton, C. (1998) Preventing conduct problems in Head Start children: strengthening parenting competencies. Journal of Consulting and Clinical Psychology, 66, 715-730.

Westman, A. \& Garralda, M. E. (1996) Mental health promotion for young adolescents in primary care: a feasibility study. British Journal of General Practice, 46, 317.

Wickberg, B. \& Hwang, P. C. (1996) Counselling of postnatal depression: A controlled study on a population based Swedish sample. Journal of Affective Disorders, 39, 209-216.

\section{Multiple choice questions}

1. Regarding prevalence of psychiatric disorders in children and adolescents:

a the prevalence of psychiatric disorder in 7- to 12-year-old GP-attenders is 1 in 10 
$\mathrm{b}$ the prevalence of psychiatric disorder in 13to 16-year-old GP-attenders is 2 in 5

$c$ the most frequent psychiatric disorder seen in pre-school GP-attenders is separation anxiety

$d$ the most frequent psychiatric disorders seen in school-age GP-attenders are emotional disorders

e the most frequent psychiatric disorders seen in school-age GP-attenders are conduct disorders.

2. Child psychiatric disorder in primary care is associated with:

a reduced attendance

b suicide risk

c reduced functional physical symptoms

d problems in peer relationships

e maternal mental health problems.

3. GP recognition of psychiatric disorder:

a has high sensitivity

b has high specificity

c is better in cases with severe disturbance

$\mathrm{d}$ is worse in families with concurrent social stressors

e usually leads to referral to child and adolescent psychiatry.

4. The children of mothers who received a 12session intervention by the GP exhibited:

a fewer fears, sleep disorders and eating problems at age 6 years

b more aggression

c no effect on their relationship with their parents at age 6 years

d fewer neurotic symptoms in females at age 26 years

e more university degrees in men at age 26 years.
5. Interventions in primary care for behavioural and psychological difficulties have been shown to:

a improve parent confidence in managing behavioural problems

b lead to a decrease in scores on behavioural questionnaires

c lead to decreased service utilisation

d have a high take-up rate by adolescents

e be associated with low user satisfaction.

\section{MCQ answers}

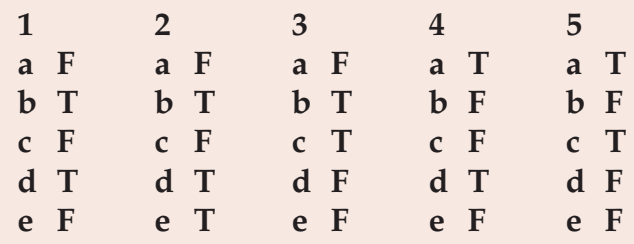

